\title{
Cellular-level understanding of supraspinal control: what can be learned from zebrafish?
}

\author{
Joanna YN Lau ${ }^{1}$, Isaac H Bianco ${ }^{1 \$}$ and Kristen E Severi ${ }^{2 \$}$ \\ ${ }^{1}$ Department of Neuroscience, Physiology \& Pharmacology, University College London, London, \\ WC1E 6BT, United Kingdom \\ ${ }^{2}$ Federated Department of Biological Sciences, New Jersey Institute of Technology, University \\ Heights, Newark, NJ, 07102, USA
}

\$Correspondence: IHB: i.bianco@ucl.ac.uk, KES: severi@njit.edu

Keywords: MLR, brainstem, locomotion, supraspinal control, reticulospinal Highlights:

- Regions such as the MLR were discovered using electrical stimulation

- Genetic and optogenetic techniques are now revealing functional heterogeneity within brainstem nuclei

- Specific neurotransmitter identity has been linked to distinct functional roles

- We review advances across vertebrate models in understanding supraspinal control of locomotion

\section{Abstract}

Electrical stimulation of various brainstem nuclei has revealed sites capable of initiating and modulating locomotion. However, subsequent experiments have revealed that these regions are functionally heterogeneous. Modern techniques including molecular-genetic methods to label cell types and optogenetic approaches to manipulate neural activity are now enabling functional examination at the cellular level. In this review, we will highlight recent work revealing distinct roles for neurons of defined neurotransmitter identity and show how comparisons across species can stimulate new experimental and analytical approaches to further our understanding of how supraspinal populations function at a cellular level to control precise aspects of behaviour.

\section{Introduction}

Major brain regions responsible for locomotor control have been discovered by detecting spinal locomotor activity following pharmacological activation or electrical stimulation of different brain sites. One such site is the mesencephalic locomotor region (MLR), first discovered in cats [1]. The MLR is defined as a site at which the intensity of electrical stimulation is proportional to the strength of induced locomotion, but which lacks direct projections to the spinal cord. Such a region has since been identified in numerous vertebrate species including rats [2], salamanders [3], lamprey [4], and carp [5], establishing it as a conserved functional element in vertebrate locomotor control. Over the past few decades, subsequent studies have identified different sub-regions constituting the mammalian MLR, namely the cuneiform nucleus ( $\mathrm{CnF}$ ) and the pedunculopontine nucleus (PPN) [2]. These regions themselves are complex, composed of neurons of different neurotransmitter identity and receiving varied sources of afferent input from different brain regions (Figure $1 A ;[6,7])$. Our growing understanding of these structural intricacies is now motivating research into what motor 
instructions these separate neural populations might provide, and how this functional organisation compares across species. Such efforts are now possible due to technical advances that allow researchers to target specific neuronal groups for monitoring or manipulation independently from surrounding regions.

\section{Functional architecture of the MLR}

With the advent of viral and genetic techniques, particularly the use of Cre-inducible viruses, it is now possible to examine the functional role of different neurotransmitter populations within the mammalian MLR [8]. A recent study used this technique to express the optogenetic activator channelrhodopsin (ChR2) specifically in either glutamatergic, GABAergic or cholinergic neurons in the mouse MLR (Figure 1A; [9]). Selective photostimulation of glutamatergic MLR neurons could evoke locomotion with speeds similar to those produced by unrestricted electrical MLR stimulation. However, optogenetic stimulation of either GABAergic or cholinergic MLR cells produced no effects when the mouse was stationary, but did cause deceleration or acceleration respectively, when the mouse was running. The role of glutamatergic MLR neurons in locomotion is consistent with other experiments specifically stimulating these neurons [10], however the identification of specific cell types within the MLR that can exert opposing effects on speed was not apparent from earlier regionwide electrical stimulation. This neurotransmitter-level examination using optogenetic targeting indicates how precise motor instructions can be attributed to particular neuronal subtypes.

Building upon the finding that glutamatergic MLR neurons can initiate locomotion $[9,10]$, two studies have recently identified distinct locomotor functions for glutamatergic cells in the CnF and PPN. These cell groups were targeted by localised viral injections and optical fibre placement. Josset et al. used optogenetic stimulation to specifically attribute initiation of locomotion to glutamatergic $\mathrm{CnF}$ neurons, but not those in the PPN [11]. In contrast, Caggiano et al. found that optogenetic stimulation of glutamatergic PPN neurons could also elicit locomotion, but only with high frequency stimulation [12]. Notably, the two glutamatergic populations do not show equal effects on locomotion initiation; PPN stimulation induced locomotion with longer latency and reduced speeds as compared to glutamatergic CnF stimulation. These two studies additionally used optogenetic [11] and chemogenetic [12] inhibition to demonstrate a requirement for glutamatergic $\mathrm{CnF}$ cells for high speed locomotion and glutamatergic PPN cells for low speed locomotion. Because such direct manipulations may not represent the activity of these regions in the course of normal physiology, Caggiano et al. conducted extracellular recordings from these separate glutamatergic groups, verifying the presence of high-speed selective neurons in the $\mathrm{CnF}$, and low-speed selective neurons in the PPN. Furthermore, by characterising the differing connectivity of the CnF and PPN, Caggiano and colleagues were able to hypothesise how these distinct behaviours could arise from separate neural pathways. These studies illustrate the functional diversity that can be found through progressively focussed investigation of individual cell groups (Figure 1B).

\section{Brainstem structural and functional diversity}

Attributing specific functional roles to neuronal subtypes can also be applied to supraspinal circuits downstream of the MLR. In mammals, the gigantocellular nuclei of the medial reticular formation receive inputs from the MLR [13], and within these nuclei there exist multiple neuronal populations with distinct neurotransmitter expression [14]. A recent study found that while optogenetic stimulation of the entire lateral paragigantocellular nucleus (LPGi) failed to induce or modulate locomotion, selective viral targeting revealed dramatically opposing effects of specific cell types [15]. 
Optogenetic photoactivation of glutamatergic LPGi neurons initiated locomotion in mice, whilst a similar stimulation of glycinergic LPGi neurons produced locomotor arrest. Neurons promoting immobility have also been recently described in the rostral medulla of mice [16]. In this case, optogenetic stimulation of genetically targetable glutamatergic V2a neurons halts ongoing locomotion. Thus, as in the MLR, targeting of distinct cell types is starting to delineate specific and opposing functions within supraspinal control regions. Supraspinal regions containing mixtures of neurons with distinct physiological roles is likely to be a conserved feature across species: recent experiments in lamprey have identified brainstem neurons related to swim cessation located amongst cells associated with initiating and maintaining locomotion [17].

\section{Insights from zebrafish: linking identifiable brainstem neurons to specific locomotor manoeuvres}

Larval zebrafish provide a model system where it is possible to come close to understanding the relationship between the single cells within supraspinal populations and precise aspects of behaviour. In this model organism, reticulospinal neurons are highly stereotyped and many are uniquely identifiable across individual animals [18]. In addition, larval zebrafish perform a range of kinematically distinct swim bout types, which are deployed in particular behavioural contexts [19], allowing researchers to investigate a multitude of behaviours and detailed kinematics beyond the initiation and cessation of locomotion. Due to the optical transparency of larval zebrafish, experimenters have also been able to conduct in vivo calcium imaging of reticulospinal populations during execution of these behaviours, or during presentation of well-defined sensory stimuli which evoke specific swim types.

In a pioneering study, Gahtan and colleagues performed calcium imaging of the larval zebrafish reticulospinal population during tap-evoked escape responses [20]. Subsequent studies have used calcium imaging to monitor reticulospinal activity in relation to sensory stimuli that reliably induce particular behaviours [21-23], or alongside direct recording of specific swim patterns $[24,25]$. Furthermore, by rigorously characterising behaviour and testing the role of functionally identified cells using precise cell ablations or stimulation, individual neurons in the larval zebrafish hindbrain have successfully been linked with the generation of specific kinematic features such as turn amplitude, tail posture, and swimming speed [24-26] (Figure 2A).

Targeted electrophysiological recordings from identifiable neurons are a powerful means to examine connectivity within hindbrain motor control circuits. A recent study used whole cell recordings to establish the connectivity of glycinergic feedforward (FF) inhibitory neurons within the Mauthner escape circuit of larval zebrafish [27]. By making use of anatomical neurotransmitter patterning [28] (Figure 2B), circuit modelling, laser ablations and behavioural assays, FF neurons were shown to function within a circuit motif that establishes left-right escape direction and prevents maladaptive bilateral activity in response to ambiguous sensory stimuli.

Larval zebrafish also provide opportunities for minimally biased identification of neural circuits related to specific features of locomotion, throughout the brainstem. Such studies can benefit from detailed characterisation of the molecular phenotypes of hindbrain neurons [29], the creation of specific driver lines [30], and the establishment of open-access community brain atlases [31-33]. A recent study by Severi and colleagues utilised these tools to describe the neurotransmitter phenotype and transcription factor expression of hindbrain neurons active during motor output or in response to visual stimulation [34]. One group of swim-related cells were identified as glycinergic and engrailed $1 b$ positive, setting the stage for future experiments to determine exactly how these 
inhibitory cells influence behaviour. Community brain atlases that allow researchers to aggregate functional and anatomical data across experiments are becoming increasingly powerful tools for multiple model species [35-37].

\section{Concluding remarks}

Modern viral and genetic techniques have provided researchers with the opportunity to explore the roles of distinct neuron groups in behavioural control. Examining individual cell groups has allowed researchers to attribute specific functions to these groups that were otherwise masked through nonspecific stimulation of brain areas. This has revealed subtle and even contrasting effects on locomotion between separate cell groups within canonical regions such as the MLR, as well as in downstream populations. Research in small model organisms such as larval zebrafish provide a system where it is possible to examine supraspinal control and the single-cell level. Recent zebrafish work illustrates the importance of detailed characterisation of the features of the motor output being examined and the advantages of combining genetic, electrophysiological, and optical techniques.

For future experiments it will be important to consider other cell categories beyond neurotransmitter type, especially as some cells display a change in neurotransmitter identity over time as a form of plasticity in response to variations in physiological conditions [38,39]. Distinct cell types can be characterised based on anatomical position, connectivity, morphology and gene expression patterns. Investigation of supraspinal circuits will benefit from the use of open-access brain atlases, which will allow researchers to directly compare data. Developments in advanced optical techniques such as combined volumetric imaging and optogenetics [40], and online, "closedloop", all-optical circuit manipulation [41,42], provide the exciting opportunity to functionally identify and causally test the roles of individual neurons and groups during behaviour in different vertebrate species.

\section{Figure Legends}

Figure 1. Cellular-level understanding of supraspinal control, exemplified by functional diversity within the MLR.

A) Sagittal view of the mouse brain highlighting supraspinal regions featured in this review. Inset illustrates the locations and neurotransmitter diversity of nuclei within the MLR. CnF, cuneiform nucleus; PPN, pedunculopontine nucleus; LPGi, lateral paragigantocellular nuclei.

B) Schematic summarising the effects of stimulating different types of MLR neuron on locomotor behaviour.

Figure 2. Summary of larval zebrafish supraspinal organisation.

A) Schematic of a larval zebrafish brain with reticulospinal neurons outlined in black. Individual reticulospinal cells (coloured) have been associated with specific kinematic features including swim speed (nMLF, blue) and turning amplitude (ventromedial cells, green). 
B) Cross section at rostro-caudal level indicated in 5A. Neurons are organised in "stripes" with distinct patterns of neurotransmitter and transcription factor expression. Adapted from Kinkhabwala et al., 2011 [29].

\section{Annotated references}

**Capelli et al., 2017

In this elegant paper, mammalian brainstem populations with conflicting roles were disentangled by optogenetically isolating excitatory and inhibitory subpopulations.

**Caggiano et al., 2018

Caggiano and colleagues use optogenetics and chemogenetics to show that glutamatergic neurons in the PPN and $\mathrm{CnF}$ contribute to slow and high-speed locomotion respectively.

*Josset et al., 2018

In this work, the authors used optogenetic stimulation to determine the initiation of locomotion was localised specifically to glutamatergic $\mathrm{CnF}$ neurons, whereas glutamatergic and cholinergic neurons in the PPN contribute to other aspects of locomotion in freely behaving mice.

*Severi et al., 2018

Using large scale calcium imaging and open-access brain atlases, the authors mapped locomotionrelated active regions to specific inhibitory neurotransmitter and transcription factor groups in the zebrafish hindbrain.

*Dal Maschio et al., 2017

Using a sophisticated combination of techniques, Dal Maschio and colleagues combine simultaneous 3D stimulation of multiple targeted neurons, volumetric functional imaging, and behavioural tracking to investigate circuit function in larval zebrafish. 


\section{References}

1. Shik ML, Severin F V, Orlovsky GN: Control of walking and running by means of electrical stimulation of the mesencephalon. Electroencephalogr Clin Neurophysiol 1969, 26:549.

2. Skinner RD, Garcia-Rill E: The mesencephalic locomotor region (MLR) in the rat. Brain Res 1984, 323:385-9.

3. Cabelguen J-M, Bourcier-Lucas C, Dubuc R: Bimodal locomotion elicited by electrical stimulation of the midbrain in the salamander Notophthalmus viridescens. J Neurosci 2003, 23:2434-9.

4. Sirota MG, Di Prisco GV, Dubuc R: Stimulation of the mesencephalic locomotor region elicits controlled swimming in semi-intact lampreys. Eur J Neurosci 2000, 12:4081-4092.

5. Kashin SM, Feldman AG, Orlovsky GN: Locomotion of fish evoked by electrical stimulation of the brain. Brain Res 1974, 82:41-47.

6. Garcia-Rill E: The basal ganglia and the locomotor regions. Brain Res 1986, 396:47-63.

7. Martinez-Gonzalez C, Bolam JP, Mena-Segovia J: Topographical organization of the pedunculopontine nucleus. Front Neuroanat 2011, 5:22.

8. Zhao S, Ting JT, Atallah HE, Qiu L, Tan J, Gloss B, Augustine GJ, Deisseroth K, Luo M, Graybiel AM, et al.: Cell type-specific channelrhodopsin-2 transgenic mice for optogenetic dissection of neural circuitry function. Nat Methods 2011, 8:745-52.

9. Roseberry TK, Lee AM, Lalive AL, Wilbrecht L, Bonci A, Kreitzer AC: Cell-Type-Specific Control of Brainstem Locomotor Circuits by Basal Ganglia. Cell 2016, 164:526-537.

10. Lee AM, Hoy JL, Bonci A, Wilbrecht L, Stryker MP, Niell CM: Identification of a Brainstem Circuit Regulating Visual Cortical State in Parallel with Locomotion. Neuron 2014, 83:455466.

11. Josset N, Roussel M, Lemieux M, Lafrance-Zoubga D, Rastqar A, Bretzner F: Distinct Contributions of Mesencephalic Locomotor Region Nuclei to Locomotor Control in the Freely Behaving Mouse. Curr Biol 2018, 28:884-901.e3.

12. Caggiano V, Leiras R, Goñi-Erro H, Masini D, Bellardita C, Bouvier J, Caldeira V, Fisone G, Kiehn O: Midbrain circuits that set locomotor speed and gait selection. Nature 2018, 553:455-460.

13. Noga BR, Kriellaars DJ, Brownstone RM, Jordan LM: Mechanism for activation of locomotor centers in the spinal cord by stimulation of the mesencephalic locomotor region. $J$ Neurophysiol 2003, 90:1464-78.

14. Greene RW, Carpenter DO: Actions of neurotransmitters on pontine medical reticular formation neurons of the cat. J Neurophysiol 1985, 54:520-531.

15. Capelli P, Pivetta C, Soledad Esposito M, Arber S: Locomotor speed control circuits in the caudal brainstem. Nature 2017, 551:373.

16. Bouvier J, Caggiano V, Leiras R, Caldeira V, Bellardita C, Balueva K, Fuchs A, Kiehn O: Descending Command Neurons in the Brainstem that Halt Locomotion. Cell 2015, 163:1191-1203.

17. Juvin L, Grätsch S, Trillaud-Doppia E, Gariépy JF, Büschges A, Dubuc R: A Specific Population of Reticulospinal Neurons Controls the Termination of Locomotion. Cell Rep 2016, 15:23772386. 
18. Kimmel CB, Powell SL, Metcalfe WK: Brain Neurons Which Project to the Spinal Cord in Young Larvae of the Zebrafish. J Comp Neurol 1982, 205:112-127.

19. Marques JC, Lackner S, Félix R, Orger MB: Structure of the Zebrafish Locomotor Repertoire Revealed with Unsupervised Behavioral Clustering. Curr Biol 2018, 28:181-195.e5.

20. Gahtan E, Sankrithi N, Campos JB, O'Malley DM: Evidence for a Widespread Brain Stem Escape Network in Larval Zebrafish. J Neurophysiol 2002, 87:608-614.

21. Orger MB, Kampff AR, Severi KE, Bollmann JH, Engert F: Control of visually guided behavior by distinct populations of spinal projection neurons. Nat Neurosci 2008, 11:327-33.

22. Wang $\mathrm{W}-\mathrm{C}, \mathrm{McLean} \mathrm{DL}$ : Selective responses to tonic descending commands by temporal summation in a spinal motor pool. Neuron 2014, 83:708-21.

23. Bhattacharyya K, McLean DL, Maclver MA: Visual Threat Assessment and Reticulospinal Encoding of Calibrated Responses in Larval Zebrafish. Curr Biol 2017, 27:2751-2762.e6.

24. Huang KH, Ahrens MB, Dunn TW, Engert F: Spinal projection neurons control turning behaviors in zebrafish. Curr Biol 2013, 23:1566-1573.

25. Thiele TR, Donovan JC, Baier H: Descending Control of Swim Posture by a Midbrain Nucleus in Zebrafish. Neuron 2014, 83:679-691.

26. Severi KE, Portugues R, Marques JC, O'Malley DM, Orger MB, Engert F: Neural control and modulation of swimming speed in the larval zebrafish. Neuron 2014, 83:692-707.

27. Koyama M, Minale F, Shum J, Nishimura N, Schaffer CB, Fetcho JR, Bean B, Bhatt D, Otto S, Depoister $B$, et al.: A circuit motif in the zebrafish hindbrain for a two alternative behavioral choice to turn left or right. Elife 2016, 5:451-465.

28. Koyama M, Kinkhabwala A, Satou C, Higashijima S, Fetcho J: Mapping a sensory-motor network onto a structural and functional ground plan in the hindbrain. Proc Natl Acad Sci U S A 2011, 108:1170-1175.

29. Kinkhabwala A, Riley M, Koyama M, Monen J, Satou C, Kimura Y, Higashijima S-I, Fetcho J: A structural and functional ground plan for neurons in the hindbrain of zebrafish. Proc Natl Acad Sci U S A 2011, 108:1164-1169.

30. Satou C, Kimura Y, Hirata H, Suster ML, Kawakami K, Higashijima S: Transgenic tools to characterize neuronal properties of discrete populations of zebrafish neurons. Development 2013, 140:3927-3931.

31. Randlett $\mathrm{O}$, Wee CL, Naumann EA, Nnaemeka O, Schoppik D, Fitzgerald JE, Portugues R, Lacoste $A M B$, Riegler $C$, Engert $F$, et al.: Whole-brain activity mapping onto a zebrafish brain atlas. Nat Methods 2015, 12:1039-1046.

32. Marquart GD, Tabor KM, Brown M, Strykowski JL, Varshney GK, LaFave MC, Mueller T, Burgess SM, Higashijima S, Burgess HA: A 3D Searchable Database of Transgenic Zebrafish Gal4 and Cre Lines for Functional Neuroanatomy Studies. Front Neural Circuits 2015, 9.

33. Marquart GD, Tabor KM, Horstick EJ, Brown M, Geoca AK, Polys NF, Nogare DD, Burgess HA: High-precision registration between zebrafish brain atlases using symmetric diffeomorphic normalization. Gigascience 2017, 6:1-15.

34. Severi KE, Böhm UL, Wyart C: Investigation of hindbrain activity during active locomotion reveals inhibitory neurons involved in sensorimotor processing. Sci Rep 2018, 8:13615. 
35. Ma Y, Hof PR, Grant SC, Blackband SJ, Bennett R, Slatest L, McGuigan MD, Benveniste H: A three-dimensional digital atlas database of the adult $\mathrm{C} 57 \mathrm{BL} / 6 \mathrm{~J}$ mouse brain by magnetic resonance microscopy. Neuroscience 2005, 135:1203-1215.

36. Johnson GA, Badea A, Brandenburg J, Cofer G, Fubara B, Liu S, Nissanov J: Waxholm Space: An image-based reference for coordinating mouse brain research. Neuroimage 2010, 53:365-372.

37. Sunkin SM, Ng L, Lau C, Dolbeare T, Gilbert TL, Thompson CL, Hawrylycz M, Dang C: Allen Brain Atlas: an integrated spatio-temporal portal for exploring the central nervous system. Nucleic Acids Res 2013, 41:D996-D1008.

38. Bertuzzi $\mathrm{M}$, Chang $\mathrm{W}$, Ampatzis K: Adult spinal motoneurons change their neurotransmitter phenotype to control locomotion. Proc Natl Acad Sci 2018, 115:E9926-E9933.

39. Dulcis D, Lippi G, Stark CJ, Do LH, Berg DK, Spitzer NC: Neurotransmitter Switching Regulated by miRNAs Controls Changes in Social Preference. Neuron 2017, 95:1319-1333.e5.

40. dal Maschio M, Donovan JC, Helmbrecht TO, Baier H: Linking Neurons to Network Function and Behavior by Two-Photon Holographic Optogenetics and Volumetric Imaging. Neuron 2017, 94:774-789.e5.

41. Zhang Z, Russell LE, Packer AM, Gauld OM, Häusser M: Closed-loop all-optical interrogation of neural circuits in vivo. Nat Methods 2018, 15:1037-1040.

42. Vladimirov N, Wang C, Höckendorf B, Pujala A, Tanimoto M, Mu Y, Yang C-T, Wittenbach JD, Freeman J, Preibisch S, et al.: Brain-wide circuit interrogation at the cellular level guided by online analysis of neuronal function. Nat Methods 2018, 15:1117-1125. 
A

Glutamatergic cells

$\Delta$ GABAergic cells

- Cholinergic cells
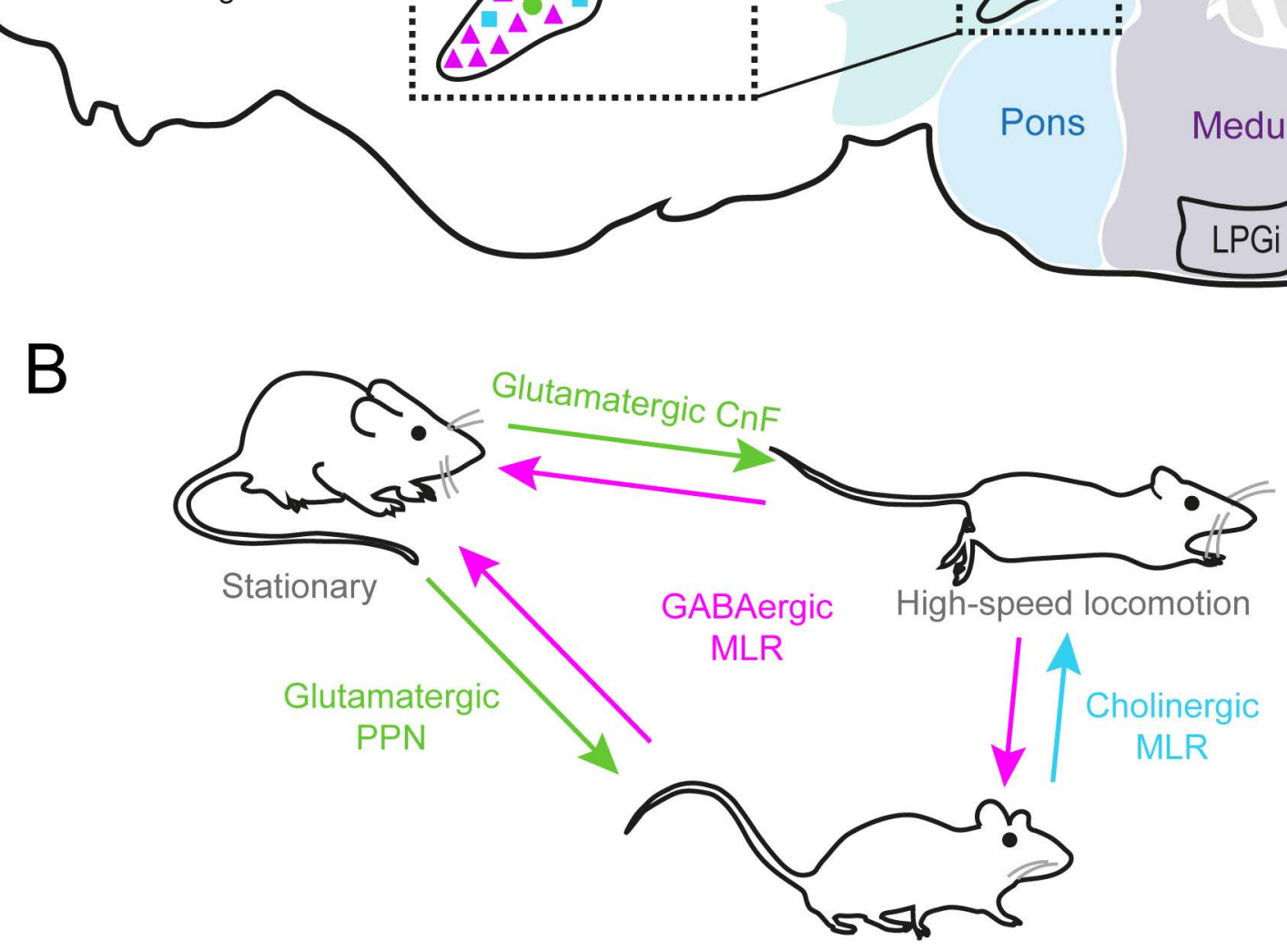

Low-speed locomotion 
A

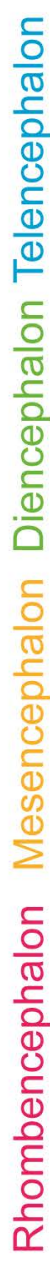

B

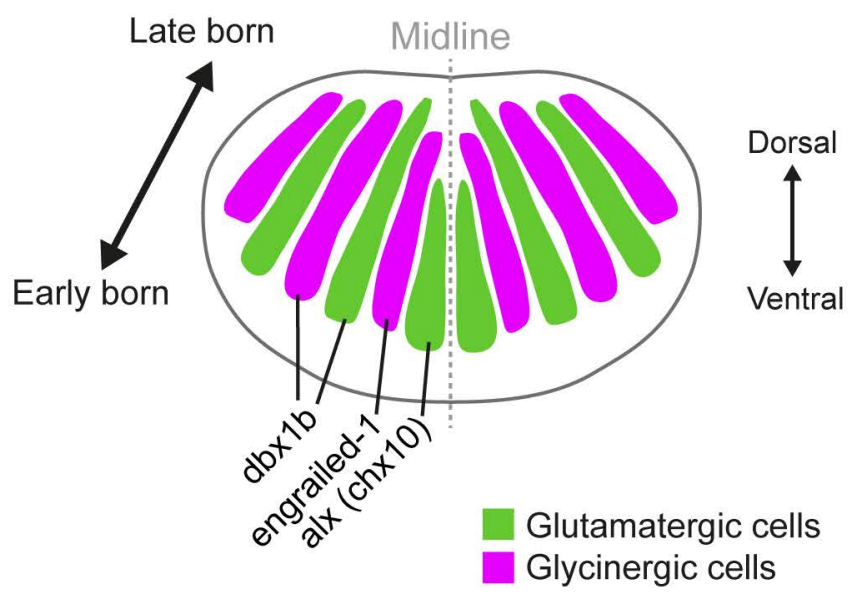

